\title{
Landscape of somatic mutations in gastric cancer assessed using next-generation sequencing analysis
}

\author{
XUAN PAN $^{1 *}$, XIAOZHI JI ${ }^{1,2^{*}}$, RENMIN ZHANG ${ }^{1,2}$, ZHAOFEI ZHOU $^{1}$, YUEJIAO ZHONG ${ }^{1}$, \\ WEI PENG ${ }^{1}$, NING SUN ${ }^{1}$, XINYU XU ${ }^{3}$, LEI XIA ${ }^{3}$, PANSONG LI ${ }^{4}$, JIANWEI LU ${ }^{1}$ and JING TU ${ }^{5}$ \\ ${ }^{1}$ Department of Medical Oncology, Jiangsu Cancer Hospital, Jiangsu Institute of Cancer Research, \\ Nanjing Medical University Affiliated Cancer Hospital, Nanjing, Jiangsu 210009; ${ }^{2}$ Xuzhou Medical University, \\ Xuzhou, Jiangsu 221004; ${ }^{3}$ Department of Pathology, Jiangsu Cancer Hospital, Jiangsu Institute of Cancer Research, \\ Nanjing Medical University Affiliated Cancer Hospital, Nanjing, Jiangsu 210009; ${ }^{4}$ Department of Research \\ and Development, Geneplus-Beijing Institute, Beijing 102206; ${ }^{5}$ State Key Laboratory of Bioelectronics, \\ School of Biological Science and Medical Engineering, Southeast University, Nanjing, Jiangsu 210096, P.R. China
}

Received January 8, 2018; Accepted June 7, 2018

DOI: $10.3892 / \mathrm{ol} .2018 .9314$

\begin{abstract}
Gastric cancer is a highly heterogeneous disease and the second leading cause of cancer-associated mortality. However, the genomic basis of gastric cancer is not completely understood and the underlying genetic heterogeneity has not been well studied. In the present study, 1,021 genes were sequenced and the somatic mutations of 45 formalin-fixed, paraffin-embedded gastric adenocarcinoma samples were assessed using next-generation sequencing technologies. In the present study, a median sequencing coverage depth of 708-fold was achieved. Somatic genomic alterations were detected in $37 / 45$ patients $(82.4 \%)$ and the most frequent genetic alterations identified were tumor protein P53 (TP53) gene mutations. Mutations in MLL4, ERBB3, FBXW7, MLL3, MTOR, NOTCH1, PIK3CA, KRAS, ERBB4 and EGFR were also detected. Patients with TP53 mutations had a higher number of somatic mutations, and the total number of somatic mutations was weakly correlated with patient age. These results provided data on the intratumoral heterogeneity of gastric cancer and may be used in order to develop personalized cancer therapy.
\end{abstract}

Correspondence to: Dr Jianwei Lu, Department of Medical Oncology, Jiangsu Cancer Hospital, Jiangsu Institute of Cancer Research, Nanjing Medical University Affiliated Cancer Hospital, 42 Baiziting Road, Nanjing, Jiangsu 210009, P.R. China

E-mail:1ujw@medmail.com.cn

Dr Jing Tu, State Key Laboratory of Bioelectronics, School of Biological Science and Medical Engineering, Southeast University, 2 Sipailou, Nanjing, Jiangsu 210096, P.R. China

E-mail: jtu@seu.edu.cn

${ }^{*}$ Contributed equally

Key words: tumor protein p53 mutation, gastric cancer, somatic mutation, next-generation sequencing, sequencing

\section{Introduction}

Gastric cancer is the second leading cause of cancer-associated mortality globally (1). The incidence of gastric cancer is particularly high in Eastern Asian countries, including China, Japan and Korea, as well as in South America (1). Of these countries, China has the highest overall incidence (1). Gastric cancer is a highly heterogeneous disease that can be categorized into two major histological subtypes, intestinal and diffuse, on the basis of the Lauren classification $(2,3)$. It has been reported that genomic or molecular classifications are important for evaluating the curative effects and prognosis of gastric cancer (4). Aberrant genetic and molecular changes occur during oncogenesis and gastric cancer progression, some of which are representative of therapeutic targets, including human epidermal growth factor receptor 2 (HER-2), MET proto-oncogene, receptor tyrosine kinase (MET) and vascular endothelial growth factor receptor (VEGFR) (5). However, despite advances in targeted therapy, the curative effect of treatments has not improved (6). When developing targeted therapies for gastric cancer, somatic mutations that occur with low frequencies may be lost to screening as certain detection approaches have high detection limits (3).

Recent genomic studies have identified four molecular subtypes (MSS/EMT, MSI, MSS/TP53+ and MSS/TP53-) of gastric cancer that are associated with distinct patterns of disease progression and prognosis (4). The Cancer Genome Atlas (TCGA) study also reported 4 genetic subgroups (7). The chromosomal instability (CIN) classification is present in $\sim 50 \%$ of cases and is associated with intestinal morphology, as well as a high frequency and density of p53 mutations. The genomically stable (GS) classification is present in $20 \%$ of cases and is associated with diffuse histology, as well as CDH1 and RhoA mutations. A further $20 \%$ of cases are MSI and are associated with hyper mutations of somatic DNA (7). Finally, $10 \%$ of cases are associated with Epstein Barr virus and have a high frequency of PIK3CA and ARID1A mutations (7). It may be possible to illustrate the mutation pattern of 
gastric cancer through the comprehensive analysis of multiple studies, and molecular markers that may aid in guiding patient management.

Historically, mutations have been profiled using Sanger sequencing, reverse transcription-quantitative polymerase chain reaction (RT-qPCR) and microarrays. The Sanger sequencing method generates a precise base sequence, typically $<1,000$ base pairs (bp) in one sequencing run (8). As a result, only mutations within this short sequence can be detected at any one time. The heterogeneity resolution rate of Sanger sequencing is relatively high, at $15-20 \%(9,10)$. However, Sanger sequencing for a large number of genes is expensive and labor-intensive. qPCR has a higher resolution rate when it comes to analyzing low frequency mutations compared with Sanger sequencing (11). However, qPCR can only profile one locus with known alleles in each reaction, making it expensive and time-consuming for sequencing multiple genes. Microarrays are an efficient parallel profiling approach for analyzing large heterogeneous loci (12). However, the high detection limit restricts the application of microarrays for somatic mutation profiling. Next-generation sequencing (NGS) technologies are low cost with good throughput and resolution rates. NGS may therefore be used to overcome the problems associated with other sequencing methods and provide accurate information regarding gene mutations to aid in treatment decisions (13). NGS can be used to investigate multiple genes simultaneously with only small amounts of DNA that can be obtained from a variety of specimens, including formalin-fixed, paraffin-embedded (FFPE) tissues, and NGS has an improved sensitivity compared with routine technologies.

It has been reported that genetic alterations result in heterogeneous disease states in human cancer, and investigating this heterogeneity is essential for understanding the potential mechanisms of oncogenesis and developing effective treatments (14). A number of studies have reported that genetic mutations occur in oncogenes and tumor suppressor genes in gastric cancer (15-17). A number of genes, including TP53 and HER-2, have been identified as driver genes in gastric cancer (18). However, the association between somatic mutations and clinical features has not yet been completely elucidated. It is therefore important to profile the somatic mutation pattern of driver genes and potential driver genes in gastric cancer. TP53 is the most frequently mutated gene in human cancer, with alterations occurring in $\sim 50 \%$ of all cases of cancer (19). TP53 serves a fundamental role in regulating cellular processes involved in the inhibition of proliferation, and maintaining genome integrity and stability (20). TP53 mutations have been reported in gastric cancer, suggesting that it may be an effective biomarker for this disease $(4,21,22)$. However, the mutation patterns of TP53 have been demonstrated to differ within the same tumor sample, and intratumoral heterogeneity exists in TP53-inactivation mechanisms (3). The association between TP53 mutation patterns and clinical features is obscure and it is unknown whether TP53 mutations influence the other genes. Using whole-exome sequencing, it was demonstrated that inactivating mutations in cell adhesion and chromatin remodeling genes are also frequent (16).

To improve our understanding of the genomic basis of gastric cancer and to identify the underlying genetic heterogeneity, NGS technologies were used to screen genetic mutations in potential driver genes from 45 FFPE gastric adenocarcinoma samples. In total, 1,021 cancer-related genes, including certain known oncogenes and tumor suppressor genes, were sequenced with a median sequencing coverage depth of 708 -fold. Over $80 \%$ of the samples were demonstrated to exhibit at least one somatic genomic alteration. In the present study, TP53 was the most frequently mutated gene, and patients with TP53 mutations had a significantly higher number of mutations.

\section{Materials and methods}

Patients and tissue sample collection. A total of 45 patients with gastric cancer were recruited from the Department of General Surgery of Nanjing Medical University Affiliated Cancer Hospital (Nanjing, China) between January 2013 and December 2013 (39 males and 6 females). The median age at diagnosis was 60 years (range, 32-79 years). All patients provided written informed consent and were diagnosed using histology. The present study was approved by the Ethics Committee of Jiangsu Cancer Hospital (Nanjing, China).

A total of 45 human gastric cancer tissues were analyzed using target capture and NGS. Germ-line DNA from matched adjacent non-cancerous tissues was used as a reference sequence to detect somatic alterations.

Gastric tumor specimens were harvested from three anatomical locations: 15 from the antrum (33.33\%), 19 from the body $(42.22 \%)$ and 11 from the cardia $(24.44 \%)$. At least two senior pathologists from the department of Pathology, Jiangsu Cancer Hospital (Nanjing, China), reviewed all histopathological diagnoses independently. In the present study, 14 (31.11\%) cases were classified as diffuse-type, $26(57.78 \%)$ as intestinal-type, and $5(11.11 \%)$ as mixed intestinal and diffuse histology. The clinical stage was determined according to 7th National Comprehensive Cancer Network guidelines (23). Of the 45 samples collected, 5 (11.11\%) were determined to be stage I, 6 (13.33\%) were stage II, and 34 (75.56\%) were stage III. Patients had a median follow-up time of 39 months.

DNA extraction and target capture sequencing. Human gastric cancer tissues were fixed in $10 \%$ formalin up to $24 \mathrm{~h}$ at room temperature. Genomic DNA was isolated from FFPE tumor samples and matched non-cancerous tissues using the QIAamp DNA FFPE Tissue kit (Qiagen GmbH, Hilden, Germany), according to the manufacturer's protocol. The DNA concentration was measured using the Qubit dsDNA HS (High Sensitivity) assay kit in the Qubit fluorometer (Invitrogen; Thermo Fisher Scientific, Inc., Waltham, MA, USA). To test the DNA integrity, 200 ng extracted DNA was loaded onto the $1 \%$ agarose gel with $\lambda$-Hind III digest DNA marker (Takara Biotechnology Co., Ltd., Dalian, China). The DNA samples that were longer than the second largest bonds $(9,416 \mathrm{bp})$ of $\lambda$-Hind III digest DNA marker were considered as integrated samples and were used for subsequent analysis.

A panel was designed, including exons of 1,017 genes, as well as introns, promoters and fusion regions of 24 genes. In brief, the panel comprised recurrent mutated genes in gastric cancer as recorded in the COSMIC database (http://cancer. sanger.ac.uk/cosmic), oncogenes and tumor suppressor genes associated with tumorigenesis and metastasis in gastric cancer $(16,24)$, and genes associated with other cancer types 

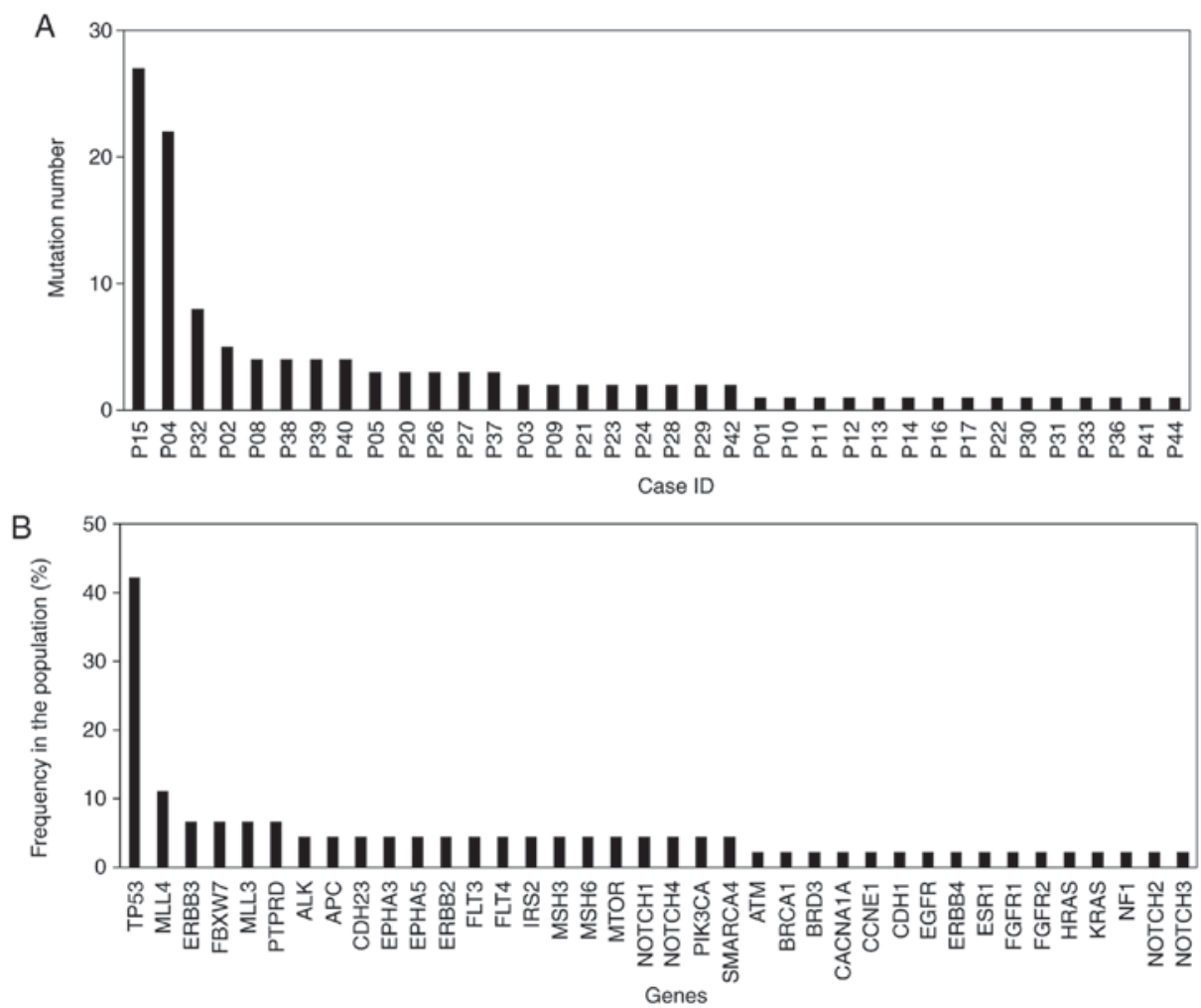

Figure 1. Mutation numbers and frequencies in 45 gastric cancer specimens. (A) Number of mutations per sample. (B) Frequencies of mutated genes in this cohort.

as recorded in the TCGA network (https://cancergenome.nih. gov/).

Library constructions were prepared using protocols recommended in the Illumina TruSeq DNA Library Preparation kit (Illumina, Inc., San Diego, CA, USA) using $1 \mu \mathrm{g}$ DNA isolated from FFPE tumor samples. DNA was sheared prior to using an ultrasonoscope with a peak of $250 \mathrm{bp}$, followed by end repair. Fragments were then ligated to the Illumina-indexed adapters according to the standard library construction protocol. Custom-designed biotinylated oligonucleotide probes (Roche NimbleGen, Inc., Madison, WI, USA) covering $\sim 1.1 \mathrm{M}$ bp of 1,021 genes were used to capture target sequences in the libraries. DNA sequencing was performed with 2 x150 bp paired-end reads on the HiSeq 3000 sequencing system (Illumina, Inc.).

Sequencing data analysis. Terminal adaptor sequences and low-quality reads were removed from the raw data. The clean reads were aligned to the human genome build GRCh37 using BWA software version 0.7.12-r1039 (25,26). Somatic single nucleotide variants (SNVs) and somatic small insertions and deletions (Indels) were generated using MuTect version 1.1.4 (27) and GATK version 3.4-46-gbc02625 (28), respectively. Candidate somatic mutations were SNVs and Indels where the variant allele fraction (VAF) was $\geq 2 \%$ and there were $\geq 5$ high-quality reads (Phred score $\geq 30$, mapping quality $\geq 13$, and without paired-end reads bias) containing the target base. The candidate mutations were annotated to genes using ANNOVAR software (29) to identify the mutated protein-coding position and exclude intronic and silent changes. Missense, nonsense, frameshift, spans, splicing, cds-del, cds-ins, stop-gain and stop-loss mutations were retained. CONTRA v2.0.8 (30) was used to detect copy number variants (CNVs) and a manual visual inspection step was performed to further remove artifactual changes.

Statistical analysis of clinical and genetic data. All statistical analyses were performed using SPSS version 19.0 (IBM Corp., Armonk, NY, USA). Unpaired Student's t-tests and $\chi^{2}$ tests were used to compare the mutation status of TP53 genes between different groups with different clinical and pathological characteristics. Mann-Whitney U tests were used to compare the overall number of mutations between the group of TP53-WT (wild-type) and TP53-Mut (mutated type). The mutation number data are presented as the mean \pm interquartile range. Pearson's correlation analysis was used to evaluate the correlation between the number of mutations and patient age. The statistic of overall survival (OS) were carried out using the Kaplan-Meier method, and differences between groups were evaluated using the log-rank test. To determine which independent factors had a significant impact on OS, Cox proportional hazards regression analysis was performed. $\mathrm{P}<0.05$ was considered to indicate a statistically significant difference.

\section{Results}

Target capture and NGS of human gastric cancer. In the present study, a panel of 1,021 genes was investigated. A median sequencing coverage depth of 708 -fold (from 14- to 1,609-fold) was achieved for 45 gastric cancer tissues. At least one somatic genomic alteration, including SNVs, Indels or CNVs, was detected in 37/45 (82.4\%) patients. The maximum number of mutations identified in one tumor sample was 27 
(Fig. 1A). There were a total of 121 mutations in this cohort and the highest mutation rate was $73.4 \%$ (TP53 p.R273C; Fig. 1B). Furthermore, somatic mutations in the TP53 gene were the most frequently identified genetic alterations, occurring in 19/45 (42.2\%) samples. Somatic mutations in the MLL4 gene were the second most frequent, occurring in 5/45 (11.1\%) samples (Fig. 1B). Mutations were identified in the ERBB3, FBXW7 and MLL3 genes in more than one case. Finally, six other mutated genes (MTOR, NOTCH1, PIK3CA, KRAS, ERBB4 and EGFR) were identified in one case each (Fig. 1B). CNVs were detected in 8 patients and ten genes (Table I). CNVs of ERBB2, which was the most frequent gene, occurred in 3 patients.

In the coding regions, the average number of mutations per sample was 3 (mutation range, 1-27). The somatic SNVs list is presented in Fig. 2A. The most frequently occurring variant was the percentage of $\mathrm{C}>\mathrm{T}$ across the coding regions, at $52.3 \%$. The number of SNVs per patient genome varied greatly (Fig. 2B).

TP53 mutations are associated with clinicopathological subtypes of gastric cancer. TP53 was the most frequently mutated gene, with 12 missense, 4-frame shift, 2 non-sense and 1 splice-5 mutation (Table II). Changes in TP53 protein are summarized in Fig. 3. Mutational hotspots were identified in codons 91, 132, 146, 155, 156, 173, 176, 179, 195, 236, 244, 237, 273, 294 and 343. TP53 mutation rates were then compared with the clinicopathological subtypes of GC. Age, sex, histology, Lauren subtype, differentiation, venous and lymphatic invasion, staging and lymph node, liver, peritoneal and other distant metastases were risk factors included in this analysis. Two main trends were observed: i) The patients with TP53 mutations had a higher overall number of mutations (Fig. 4A); and ii) the number of mutations was significantly associated with patient age $(\mathrm{P}=0.0223$, Fig. $4 \mathrm{~B})$.

Although there was no evidence to suggest that TP53 mutation positivity was associated with age, sex, histology, Lauren subtype, differentiation, staging or distant metastasis, patients with TP53 mutations exhibited less venous invasion (10.5 vs. 46.2\%; $\mathrm{P}=0.011$ ), less perineural invasion (47.4 vs. $88.8 \%$; $\mathrm{P}=0.019)$ and less severe lymph node metastasis (N0-2 vs. N3-4; 15.8 vs. $46.2 \%$; $\mathrm{P}=0.033$; Table III). It was also reported that patients with the TP53 mutation had an improved overall survival ( $\mathrm{P}=0.109$; data not shown). However, these results are contradictory to those of previous reports $(22,31)$.

\section{Discussion}

In the present study, genomic alterations in 45 gastric cancer samples were detected using target capture and NGS analysis. Somatic mutations were detected in $82.4 \%$ of patients, indicating that gastric cancer is a highly heterogeneous disease. $\mathrm{C}: \mathrm{G}$ to $\mathrm{T}: \mathrm{A}$ transitions were more common than other single-nucleotide alterations. It has previously been reported that N-methyl-N'-nitro-N-nitrosoquanidine and $\mathrm{N}$-nitroso compounds identified in food are able to induce $\mathrm{C}$ to $\mathrm{T}$ transitions (32) and are considered to be gastric carcinogens. These foods such as pickles vegetables and salted meat are commonly consumed in Chinese populations, increasing the risk of gastric carcinogenesis (33). The results of the present study
Table I. Extent and distribution of copy number variants.

\begin{tabular}{llllr}
\hline $\begin{array}{l}\text { Case } \\
\text { ID }\end{array}$ & Gene & \multicolumn{1}{c}{ Transcripts } & Chromosome & $\begin{array}{c}\text { Copy } \\
\text { number }\end{array}$ \\
\hline P01 & MDM2 & NM_002392.4 & chr12 & 6.3 \\
P01 & ERBB3 & NM_001982.3 & chr12 & 3.2 \\
P01 & AURKA & NM_198433.1 & chr20 & 5.6 \\
P01 & GNAS & NM_001077488.2 & chr20 & 10.7 \\
P01 & VEGFA & NM_001025366.2 & chr6 & 4.7 \\
P14 & VEGFA & NM_001025366.2 & chr6 & 7.7 \\
P14 & EGFR & NM_005228.3 & chr7 & 5.5 \\
P16 & ERBB2 & NM_001005862.1 & chr17 & 25.6 \\
P23 & PIK3CA & NM_006218.2 & chr3 & 2.3 \\
P32 & ERBB2 & NM_001005862.1 & chr17 & 347.7 \\
P41 & ERBB2 & NM_001005862.1 & chr17 & 2.1 \\
P43 & CCNE1 & NM_001238.2 & chr19 & 7.7 \\
P44 & ERBB3 & NM_001982.3 & chr12 & 3.0 \\
P44 & STK11 & NM_000455.4 & chr19 & 1.8
\end{tabular}

Table II. Tumor protein P53 mutation type.

\begin{tabular}{lll}
\hline Case ID & Protein_Change & \multicolumn{1}{c}{ Mutation_Type } \\
\hline P02 & p.M237I & Missense_Mutation \\
P03 & p.R273C & Missense_Mutation \\
P04 & p.R273C & Missense_Mutation \\
P05 & p.R156Pfs*25 & Frame_Shift_Ins \\
P11 & p.C176F & Missense_Mutation \\
P14 & p.G244C & Missense_Mutation \\
P15 & p.E294Sfs*51 & Frame_Shift_Del \\
P17 & p.W146* & Nonsense_Mutation \\
P23 & p.E343Gfs*2 & Frame_Shift_Del \\
P24 & p.W91* & Nonsense_Mutation \\
P28 & p.Y236C & Missense_Mutation \\
P29 & & Splice_Site \\
P31 & p.T155P & Missense_Mutation \\
P32 & p.V173G & Missense_Mutation \\
P38 & p.H179R & Missense_Mutation \\
P39 & p.M237Cfs*10 & Frame_Shift_Del \\
P40 & p.R273C & Missense_Mutation \\
P41 & p.I195T & Missense_Mutation \\
P44 & p.K132R & Missense_Mutation \\
& &
\end{tabular}

supported previous reports that $\mathrm{C}$ to $\mathrm{T}$ transitions are typical of TP53 mutations in gastric carcinogenesis. Such mutations are considered to be the result of interactions between underlying genetic factors and environmental predisposing factors (32).

At the genetic level, TP53 is the most commonly mutated gene in human cancer (19). TP53 negatively regulates the cell cycle and induces DNA repair under environmental pressure, serving as a tumor suppressor gene (34). It was demonstrated that mutant TP53 acquires oncogenic properties that 


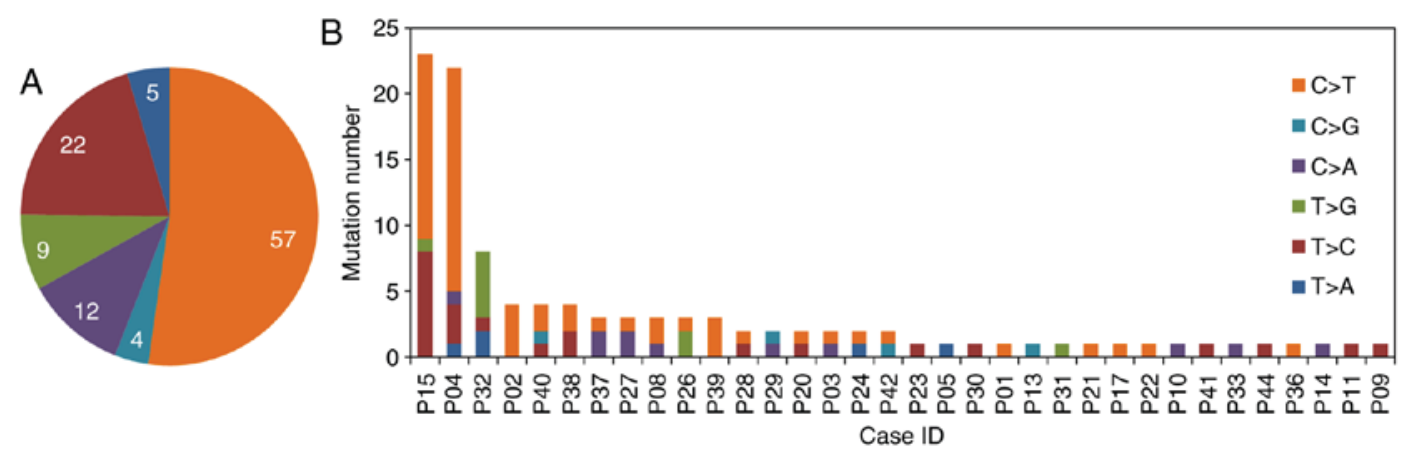

Figure 2. Mutational load and mutational signatures in 45 gastric cancer specimens. (A) Percentage of single-nucleotide variants ( $S N V ; C>T, C>G, C>A, T>C$, $\mathrm{T}>\mathrm{G}$ and $\mathrm{T}>\mathrm{A}$ ) in gastric cancer. (B) Mutational load: Stacked bar plot of the SNV count and nucleotide change with the changes indicated by different colors. Tumor samples are ordered according to the number of nucleotide variants. SNV, single nucleotide variants.

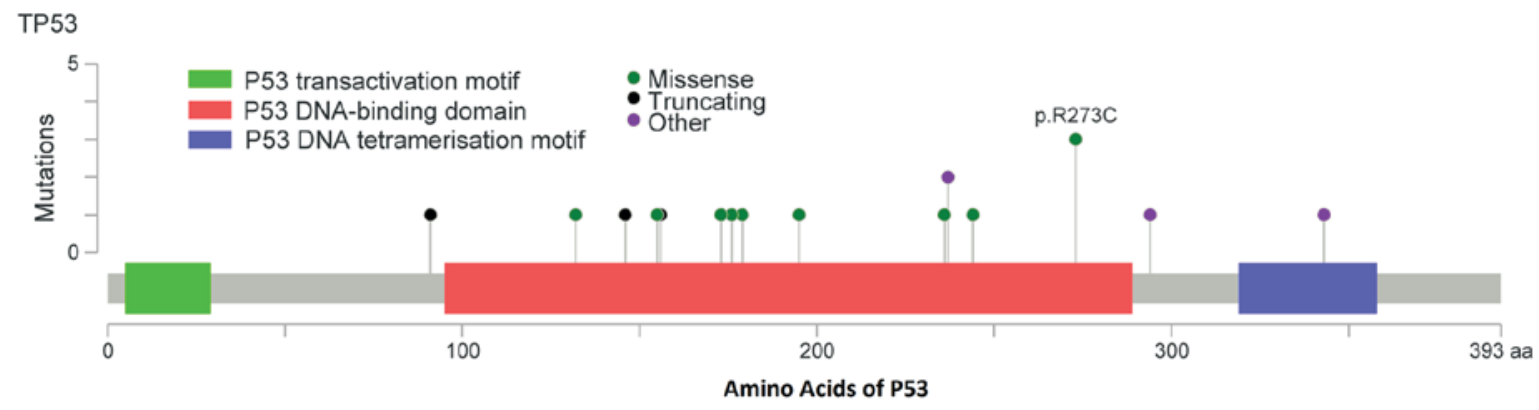

Figure 3. Mutation distribution in functional domains of tumor protein P53.
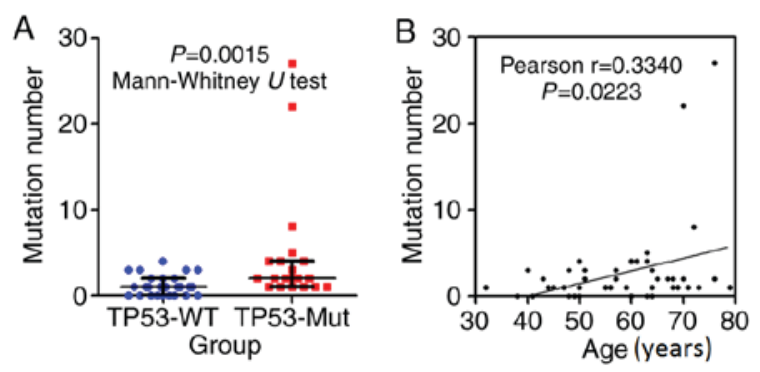

Figure 4. Association between mutation numbers, TP53 mutation and age in patients with gastric cancer. (A) Patients with a TP53 mutation had a higher number of mutations overall. The Mann-Whitney U test was used for statistical analysis. (B) The number of mutations was correlated with patient age. The Pearson correlation analysis was used for statistical analysis. WT, wild type; Mut, mutant.

are entirely independent of wild-type TP53 $(35,36)$. TP53 gain-of-function actively contributes toward various stages of tumor progression whilst also increasing resistance to anticancer treatments. It may therefore be beneficial to develop molecules that are able to recover wild-type TP53 activity and remove TP53 gain-of-function mutations from the cell. To this end, a number of studies have been performed (37-39). It is challenging to translate these approaches into a clinical setting; and a more accessible approach may be to target the downstream pathways mediating mutant TP53 activity. Progress has been made in this area, and a number of clinically approved drugs that target MET, EGFR and cholesterol synthesis pathways are already available $(6,40)$. However, further research is required.
In the present study, it was demonstrated that TP53 gene mutation was the most frequent genetic change associated with gastric cancer in a Chinese population, which is consistent with the results of previous reports $(16,41)$. According to the TCGA database, TP53 mutations occur in $36.4-55 \%$ of gastric cancer cases (42). In the present study, TP53 mutations were observed in $42.2 \%$ of gastric cancer cases, which is lower than in patients from Singapore (41), but similar to patients from Hong Kong and Japan $(22,43)$. In addition, patients with TP53 mutations had a significantly higher number of mutations overall, which suggested that the TP53 gene may serve a crucial role in the maintenance of genome integrity and stability. Zang et al have used whole-exome sequencing to identify frequent inactivating mutations in cell adhesion and chromatin remodeling genes in addition to TP53 mutations (41). TP53 has been referred to as 'the guardian of the genome' (20). Genome instability generates genetic diversity, and expedites the cell mutations and accumulation of mutated genes required to initiate tumorigenesis (44). There are conflicting results with respect to the prevalence of TP53 mutations, as well as their association with clinicopathological features in gastric cancer (45). The present study demonstrated that the patients with a TP53 mutation had less venous invasion and perineural invasion, as well as less severe lymphoid metastasis (N3-4). The role of TP53 as a biomarker for predicting prognoses in gastric cancer was also investigated. The follow-up data demonstrated that mutations in TP53 were associated with increased survival, although there was no statistical difference. We hypothesized that this may be due to the small sample size and short follow-up time. 
Table III. Patient clinicopathological characteristics between TP53 mutation+ and TP53- gastric cancer.

\begin{tabular}{|c|c|c|}
\hline Characteristic & TP53 mutation+ & TP53 mutation- \\
\hline Number & 19 & 26 \\
\hline Median age (range) & $63(47-76)$ & $55.5(32-79)$ \\
\hline \multicolumn{3}{|l|}{ Sex } \\
\hline Male & 17 & 22 \\
\hline Female & 2 & 4 \\
\hline \multicolumn{3}{|l|}{ Lauren type } \\
\hline Intestinal & 13 & 13 \\
\hline Diffuse & 4 & 10 \\
\hline Mixed & 2 & 3 \\
\hline \multicolumn{3}{|l|}{ pT stage } \\
\hline $\mathrm{T} 1$ & 2 & 1 \\
\hline $\mathrm{T} 2$ & 2 & 2 \\
\hline $\mathrm{T} 3$ & 2 & 1 \\
\hline $\mathrm{T} 4$ & 13 & 22 \\
\hline \multicolumn{3}{|l|}{ pN stage } \\
\hline No & 7 & 5 \\
\hline N1 & 5 & 3 \\
\hline N2 & 4 & 6 \\
\hline N3 & 3 & 12 \\
\hline \multicolumn{3}{|l|}{ AJCC stage (7th, ed.) } \\
\hline $\mathrm{I}$ & 3 & 2 \\
\hline II & 4 & 2 \\
\hline III & 12 & 22 \\
\hline IV & 0 & 0 \\
\hline \multicolumn{3}{|l|}{ Venous invasion } \\
\hline Positive & 2 & 12 \\
\hline Negative & 17 & 14 \\
\hline \multicolumn{3}{|l|}{ Perineural invasion } \\
\hline Positive & 9 & 21 \\
\hline Negative & 10 & 5 \\
\hline \multicolumn{3}{|l|}{ Recurrence } \\
\hline Positive & 10 & 18 \\
\hline Negative & 9 & 8 \\
\hline
\end{tabular}

TP53, tumor protein P53; T, tumor; N, node; AJCC, American Joint Committee on Cancer.

TP53, ERBB2, ERBB3, ERBB4, EGFR, MTOR and c-MET gene alterations are emerging as potential clinical biomarkers (37). Novel somatic gene targets, ARIDLA, FAT4 and MLL, are of increasing interest and importance (32). In the present study, mutations were also identified in the histone methyltransferase gene MLL3 (3/45) and the MLL4 $(5 / 45)$ gene in gastric cancer samples. MLL genes encode histone methyltransferases that are essential for the proper expression of a number of genes (46). It has been suggested that mutational and expressional alterations of MLL genes are involved in various human malignancies (47-49). Somatic mutations in the MLL3 gene have been identified in colorectal cancer (48). Amplification of the MLL4 gene has also been revealed in glioblastomas and pancreatic cancer (49). Chen et al (16) uncovered mutations in the MLL4 gene through whole-genome sequencing of two Chinese patients with gastric cancer. Je et al (50) indicated that frame shift mutations of MLL, MLL2, MLL3 and MLL5 genes and loss of expression of MLL3 protein are common in gastric cancer with high microsatellite instability (MSI-H). In the present study, novel MLL4 frame shift mutations were identified in gastric cancer. Activating signal cointegrator-2 (ASC-2) complex, named ASCOM, contains histone H3K4 methyltransferase MLL3 or its paralog MLL4 (51). Notably, in vivo and in vitro observations revealed that ASCOM acts as a crucial TP53 coactivator, and is necessary for $\mathrm{H} 3 \mathrm{~K} 4$-trimethylation and the expression of endogenous TP53 target genes in response to DNA damage (51). These findings implicated MLL3/4 in the TP53 tumor suppression pathway. Taken together, these data suggested that MLL gene mutations may contribute toward oncogenesis in gastric cancer.

In summary, NGS analysis of 1,021 genes in FFPE tissue specimens from 45 gastric adenocarcinomas was successfully performed. At least one somatic genomic alteration was detected in $37 / 45(82.4 \%)$ patients, and somatic mutations in the TP53 gene were most frequent. Patients with a TP53 mutation had a significantly higher number of mutations overall, indicating that the TP53 gene may serve a crucial role in the maintenance of genome integrity and stability. Tumor heterogeneity is a clear hallmark of gastric cancer. In the era of precision medicine, further research is required to investigate intratumoral heterogeneity, and develop an understanding of the potential mechanisms of tumorigenesis and potential therapeutic approaches.

\section{Acknowledgements}

Not applicable.

\section{Funding}

The present study was supported by the National Natural Science Foundation of China (grant no. 81502678), the 'Jiangsu Provincial Medical Youth Talent' program of the project of Invigorating Health Care through Science, Technology Education, and the Young Talents Program of Jiangsu Cancer Hospital.

\section{Availability of data and materials}

The datasets used during the current study are available from the corresponding author on reasonable request.

\section{Authors' contributions}

XP participated in study design, coordination data analysis and drafted the manuscript. XJ participated in experimental performance and coordination data analysis. RZ participated in experimental performance. ZZ, YZ, WP, and NS participated in study design. XX and LX participated in study design and reviewed all histopathological diagnoses. PL participated in coordination data analysis. JL and JT conceived of the study, 
participated in its design, revised the manuscript and made final proof. All authors read and approved the final manuscript.

\section{Ethics approval and consent to participate}

The present study was approved by the Ethics Committee of Jiangsu Cancer Hospital (Nanjing, China). The reference number of the ethics approval statement is 2013LS-6. All patients provided written informed consent to participate in this study.

\section{Patient consent for publication}

All patients provided written informed consent for publication of any associated data and accompanying images involved in this manuscript.

\section{Competing interests}

The authors declare that they have no competing interests.

\section{References}

1. Jemal A, Bray F, Center MM, Ferlay J, Ward E and Forman D: Global cancer statistics. CA Cancer J Clin 61: 69-90, 2011.

2. Hu B, El Hajj N, Sittler S, Lammert N, Barnes R and Meloni-Ehrig A: Gastric cancer: Classification, histology and application of molecular pathology. J Gastrointest Oncol 3: 251-261, 2012.

3. Wong SS, Kim KM, Ting JC, Yu K, Fu J, Liu S, Cristescu R, Nebozhyn M, Gong L, Yue YG, et al: Genomic landscape and genetic heterogeneity in gastric adenocarcinoma revealed by whole-genome sequencing. Nat Commun 5: 5477, 2014.

4. Cristescu R, Lee J, Nebozhyn M, Kim KM, Ting JC, Wong SS, Liu J, Yue YG, Wang J, Yu K, et al: Molecular analysis of gastric cancer identifies subtypes associated with distinct clinical outcomes. Nat Med 21: 449-456, 2015.

5. Deng N, Goh LK, Wang H, Das K, Tao J, Tan IB, Zhang S, Lee M, Wu J, Lim KH, et al: A comprehensive survey of genomic alterations in gastric cancer reveals systematic patterns of molecular exclusivity and co-occurrence among distinct therapeutic targets. Gut 61: 673-684, 2012.

6. Wong $\mathrm{H}$ and Yau T: Molecular targeted therapies in advanced gastric cancer: Does tumor histology matter? Therap Adv Gastroenterol 6: 15-31, 2013.

7. Cancer Genome Atlas Research Network: Comprehensive molecular characterization of gastric adenocarcinoma Nature 513: 202-209, 2014.

8. Kim Y and Yeung ES: DNA sequencing with pulsed-field capillary electrophoresis in poly(ethylene oxide) matrix Electrophoresis 18: 2901-2908, 1997.

9. Bar-Eli M, Ahuja H, Gonzalez-Cadavid N, Foti A and Cline MJ: Analysis of N-RAS exon-1 mutations in myelodysplastic syndromes by polymerase chain reaction and direct sequencing. Blood 73: 281-283, 1989.

10. Collins SJ, Howard M, Andrews DF, Agura E and Radich J: Rare occurrence of N-ras point mutations in Philadelphia chromosome positive chronic myeloid leukemia. Blood 73: 1028-1032, 1989.

11. Gentle A, Anastasopoulos F and McBrien NA: High-resolution semi-quantitative real-time PCR without the use of a standard curve. BioTechniques 31: 502, 504-506, 508, 2001.

12. Schena M, Shalon D, Heller R, Chai A, Brown PO and Davis RW: Parallel human genome analysis: Microarray-based expression monitoring of 1000 genes. Proc Natl Acad Sci USA 93: 10614-10619, 1996.

13. Meyerson M, Gabriel S and Getz G: Advances in understanding cancer genomes through second-generation sequencing. Nat Rev Genet 11: 685-696, 2010.

14. Andrechek ER, Cardiff RD, Chang JT, Gatza ML, Acharya CR, Potti A and Nevins JR: Genetic heterogeneity of Myc-induced mammary tumors reflecting diverse phenotypes including metastatic potential. Proc Natl Acad Sci USA 106: 16387-16392, 2009
15. Lin $\mathrm{Y}, \mathrm{Wu} \mathrm{Z}, \mathrm{Guo} \mathrm{W}$ and $\mathrm{Li} \mathrm{J}$ : Gene mutations in gastric cancer: A review of recent next-generation sequencing studies. Tumour Biol 36: 7385-7394, 2015.

16. Chen K, Yang D, Li X, Sun B, Song F, Cao W, Brat DJ, Gao Z, $\mathrm{Li} \mathrm{H}$, Liang H, et al: Mutational landscape of gastric adenocarcinoma in Chinese: Implications for prognosis and therapy. Proc Natl Acad Sci USA 112: 1107-1112, 2015.

17. Xu Z, Huo X, Ye H, Tang C, Nandakumar V, Lou F, Zhang D, Dong H, Sun H, Jiang S, et al: Genetic mutation analysis of human gastric adenocarcinomas using ion torrent sequencing platform. PLoS One 9: e100442, 2014.

18. Nadauld LD and Ford JM: Molecular profiling of gastric cancer: Toward personalized cancer medicine. J Clin Oncol 31: 838-839, 2013.

19. Ozaki T and Nakagawara A: p53: The attractive tumor suppressor in the cancer research field. J Biomed Biotechnol 2011: 603925 , 2011.

20. Lane DP: Cancer. p53, guardian of the genome. Nature 358: 15-16, 1992.

21. Bellini MF, Cadamuro AC, Succi M, Proença MA and Silva AE: Alterations of the TP53 gene in gastric and esophageal carcinogenesis. J Biomed Biotechnol 2012: 891961, 2012.

22. Tahara T, Shibata T, Okamoto Y, Yamazaki J, Kawamura T, Horiguchi N, Okubo M, Nakano N, Ishizuka T, Nagasaka M, et al: Mutation spectrum of TP53 gene predicts clinicopathological features and survival of gastric cancer. Oncotarget 7 : 42252-42260, 2016.

23. Ajani JA, D'Amico TA, Almhanna K, Bentrem DJ, Chao J, Das P, Denlinger CS, Fanta P, Farjah F, Fuchs CS, et al: Gastric cancer, version 3.2016, NCCN clinical practice guidelines in oncology. J Natl Compr Cancer Netw 14: 1286-1312, 2016.

24. Wang K, Yuen ST, Xu J, Lee SP, Yan HH, Shi ST, Siu HC, Deng S, Chu KM, Law S, et al: Whole-genome sequencing and comprehensive molecular profiling identify new driver mutations in gastric cancer. Nat Genet 46: 573-582, 2014.

25. Li H and Durbin R: Fast and accurate short read alignment with Burrows-Wheeler transform. Bioinformatics 25: 1754-1760, 2009.

26. 1000 Genomes Project Consortium, Abecasis GR, Altshuler D, Auton A, Brooks LD, Durbin RM, Gibbs RA, Hurles ME and McVean GA: A map of human genome variation from population-scale sequencing. Nature 467: 1061-1073, 2010.

27. Cibulskis K, Lawrence MS, Carter SL, Sivachenko A, Jaffe D, Sougnez C, Gabriel S, Meyerson M, Lander ES and Getz G: Sensitive detection of somatic point mutations in impure and heterogeneous cancer samples. Nat Biotechnol 31: 213-219, 2013.

28. McKenna A, Hanna M, Banks E, Sivachenko A, Cibulskis K Kernytsky A, Garimella K, Altshuler D, Gabriel S, Daly M and DePristo MA: The genome analysis toolkit: A mapreduce framework for analyzing next-generation DNA sequencing data. Genome Res 20: 1297-1303, 2010.

29. Wang K, Li M and Hakonarson H: ANNOVAR: Functional annotation of genetic variants from high-throughput sequencing data. Nucleic Acids Res 38: e164, 2010.

30. Li J, Lupat R, Amarasinghe KC, Thompson ER, Doyle MA, Ryland GL, Tothill RW, Halgamuge SK, Campbell IG and Gorringe KL: CONTRA: Copy number analysis for targeted resequencing. Bioinformatics 28: 1307-1313, 2012.

31. Lim BH, Soong R, Grieu F, Robbins PD, House AK and Iacopetta BJ: p53 accumulation and mutation are prognostic indicators of poor survival in human gastric carcinoma. Int $\mathrm{J}$ Cancer 69: 200-204, 1996.

32. Tatsuta M, Itoh T, Okuda S, Taniguchi $\mathrm{H}$ and Tamura H: Effect of prolonged administration of gastrin on experimental carcinogenesis in rat stomach induced by N-methyl-N'-nitro-N-nitrosoguanidine. Cancer Res 37: 1808-1810, 1977.

33. Lin SH, Li YH, Leung K, Huang CY and Wang XR: Salt processed food and gastric cancer in a Chinese population. Asian Pac J Cancer Prev 15: 5293-5298, 2014.

34. Lane D and Levine A: p53 Research: The past thirty years and the next thirty years. Cold Spring Harb Perspect Biol 2: a000893, 2010.

35. Oren M and Rotter V: Mutant p53 gain-of-function in cancer. Cold Spring Harb Perspect Biol 2: a001107, 2010.

36. Muller PA and Vousden KH: p53 mutations in cancer. Nat Cell Biol 15: 2-8, 2013.

37. Bykov VJ, Issaeva N, Shilov A, Hultcrantz M, Pugacheva E, Chumakov P, Bergman J, Wiman KG and Selivanova G: Restoration of the tumor suppressor function to mutant $\mathrm{p} 53$ by a low-molecular-weight compound. Nat Med 8: 282-288, 2002. 
38. Bykov VJN, Issaeva N, Zache N, Shilov A, Hultcrantz M, Bergman J, Selivanova G and Wiman KG: Reactivation of mutant p53 and induction of apoptosis in human tumor cells by maleimide analogs. J Biol Chem 292: 19607, 2017.

39. Lambert JM, Gorzov P, Veprintsev DB, Söderqvist M Segerbäck D, Bergman J, Fersht AR, Hainaut P, Wiman KG and Bykov VJ: PRIMA-1 reactivates mutant $\mathrm{p} 53$ by covalent binding to the core domain. Cancer Cell 15: 376-388, 2009.

40. Bang YJ, Van Cutsem E, Feyereislova A, Chung HC, Shen L, Sawaki A, Lordick F, Ohtsu A, Omuro Y, Satoh T, et al: Trastuzumab in combination with chemotherapy versus chemotherapy alone for treatment of HER2-positive advanced gastric or gastro-oesophageal junction cancer (ToGA): A phase 3, open-label, randomised controlled trial. Lancet 376: 687-697, 2010.

41. Zang ZJ, Cutcutache I, Poon SL, Zhang SL, McPherson JR, Tao J, Rajasegaran V, Heng HL, Deng N, Gan A, et al: Exome sequencing of gastric adenocarcinoma identifies recurrent somatic mutations in cell adhesion and chromatin remodeling genes. Nat Genet 44: 570-574, 2012.

42. Cerami E, Gao J, Dogrusoz U, Gross BE, Sumer SO, Aksoy BA, Jacobsen A, Byrne CJ, Heuer ML, Larsson E, et al: The cBio cancer genomics portal: An open platform for exploring multidimensional cancer genomics data. Cancer Discov 2: 401-404, 2012.

43. Wang K, Kan J, Yuen ST, Shi ST, Chu KM, Law S, Chan TL, Kan Z, Chan AS, Tsui WY, et al: Exome sequencing identifies frequent mutation of ARID1A in molecular subtypes of gastric cancer. Nat Genet 43: 1219-1223, 2011.

44. Hanahan D and Weinberg RA: Hallmarks of cancer: The next generation. Cell 144: 646-674, 2011.
45. Fenoglio-Preiser CM, Wang J, Stemmermann GN and Noffsinger A: TP53 and gastric carcinoma: A review. Hum Mutat 21: 258-270,2003.

46. Ruthenburg AJ, Allis CD and Wysocka J: Methylation of lysine 4 on histone H3: Intricacy of writing and reading a single epigenetic mark. Mol Cell 25: 15-30, 2007.

47. Parsons DW, Li M,Zhang X, Jones S, Leary RJ, Lin JC, Boca SM, Carter H, Samayoa J, Bettegowda C, et al: The genetic landscape of the childhood cancer medulloblastoma. Science 331: 435-439, 2011.

48. Sjöblom T, Jones S, Wood LD, Parsons DW, Lin J, Barber TD, Mandelker D, Leary RJ, Ptak J, Silliman N, et al: The consensus coding sequences of human breast and colorectal cancers. Science 314: 268-274, 2006

49. Huntsman DG, Chin SF, Muleris M, Batley SJ, Collins VP, Wiedemann LM, Aparicio S and Caldas C: MLL2, the second human homolog of the Drosophila trithorax gene, maps to $19 \mathrm{q} 13.1$ and is amplified in solid tumor cell lines. Oncogene 18: 7975-7984, 1999.

50. Je EM, Lee SH, Yoo NJ and Lee SH: Mutational and expressional analysis of MLL genes in gastric and colorectal cancers with microsatellite instability. Neoplasma 60: 188-195, 2013.

51. Lee J, Kim DH, Lee S, Yang QH, Lee DK, Lee SK, Roeder RG and Lee JW: A tumor suppressive coactivator complex of p53 containing ASC-2 and histone $\mathrm{H} 3$-lysine-4 methyltransferase MLL3 or its paralogue MLL4. Proc Natl Acad Sci USA 106: 8513-8518, 2009.

This work is licensed under a Creative Commons Attribution-NonCommercial-NoDerivatives 4.0 International (CC BY-NC-ND 4.0) License. 